\title{
Toward Robust Image Classification
}

\author{
Basemah Alshemali ${ }^{12}$, Alta Graham ${ }^{2}$, and Jugal Kalita ${ }^{2}$ \\ 1 Computer Science Department, College of Computer Science and Engineering, \\ Taibah University, Almadinah, KSA \\ 2 Computer Science Department, College of Engineering and Applied Science, \\ University of Colorado at Colorado Springs, Colorado Springs, USA \\ \{balshema, agraham, jkalita\}@uccs.edu
}

\begin{abstract}
Neural networks are frequently used for image classification, but can be vulnerable to misclassification caused by adversarial images. Attempts to make neural network image classification more robust have included variations on preprocessing (cropping, applying noise, blurring), adversarial training, and dropout randomization. In this paper, we implemented a model for adversarial detection based on a combination of two of these techniques: dropout randomization with preprocessing applied to images within a given Bayesian uncertainty. We evaluated our model on the MNIST dataset, using adversarial images generated using Fast Gradient Sign Method (FGSM), Jacobian-based Saliency Map Attack (JSMA) and Basic Iterative Method (BIM) attacks. Our model achieved an average adversarial image detection accuracy of $97 \%$, with an average image classification accuracy, after discarding images flagged as adversarial, of $99 \%$. Our average detection accuracy exceeded that of recent papers using similar techniques.
\end{abstract}

Keywords: Adversarial examples, Robust image classification, Bayesian uncertainty, Image cropping.

\section{Introduction}

Deep neural networks (DNN) produce excellent image classification results and are the current state-of-the-art, but have been shown to be vulnerable to attacks by adversarial examples: Images altered by the introduction of small perturbations that cause the neural network to misclassify the image [1].

Many researchers have proposed defenses against adversarial image attacks on neural network classification systems. In image preprocessing defenses, the images are altered in some way before being classified (blurring, cropping, noise) in order to disrupt any adversarial perturbations 6 6] 7n dropout randomization defenses, as the name suggests, the neural network adds randomization which supports the use of Bayesian uncertainty measurements to assess the likelihood of an image being adversarial [3] [14].

Our model uses a combination defense: We base our model on Bayesian uncertainty in a dropout neural network [17, but use a secondary defense, preprocessing, as a double-check for "edge" cases. The existence of the secondary 
defense allows us to tune the uncertainty aspect of our defense in favor of declaring "edge" images (images close to the threshold uncertainty) adversarial, with less sacrifice of clean-image accuracy than would otherwise be the case.

\section{Related Work}

Feinman et al. 3] proposed a defense based on Bayesian uncertainty with dropout, combined with kernel density estimation. They tested it with good results against adversarial examples generated via the Fast Gradient Sign Method (FGSM) [5], Basic Iterative Method (BIM) 9], Jacobian-based Saliency Map Attack (JSMA) [15], and C\&W [1] attacks. They achieved ROC-AUC scores on sets of adversarial images generated from the MNIST dataset ranging from $90.57 \%$ to $98.13 \%$ depending on attack method. This approach took advantage of the uncertainty estimates possible with dropout networks by assuming that the Bayesian uncertainty will be greater for adversarial examples than for clean data, because of the effect of the randomization on the necessarily precise perturbations. In addition, they used a Gaussian Mixture Model to analyze the outputs of the last hidden layer of their neural network, arguing that adversarial images will have a different distribution than clean ones. They also incorporated a kernel density estimate defense and evaluated their approach on MNIST [10, CIFAR-10 8], and SVHN [13] datasets.

Papernot and McDaniel [14] also presented an uncertainty based defense: A model-agnostic system in which the uncertainty is based on the predictions of a second, separate neural network which is used to train the classification network. This defense showed good results for the MNIST dataset, tested with adversarial images generated by FGSM, JSMA, and AdaDelta 2 attacks.

Several researchers have considered preprocessing based defenses against adversarial examples. Graese, Rozsa, and Boult [6] explored several preprocessing techniques with the MNIST dataset, tested with FGSM and Fast Gradient Value (FGV) [16 attacks, and found the best results with cropping and resizing: $76 \%$ and $78 \%$ accuracy for FGV and FGSM samples respectively, compared to $65 \%$ and $68 \%$ for the next best "translation" technique on their raw model. Guo et al. 7] tested cropping-resizing as well as other image transformations (image quilting, JPEG compression, etc.) and found that cropping-resizing was "very efficient" with accuracy up to $76 \%$ depending on the strength and method of attack.

\section{Methodology}

Our method uses Bayesian uncertainty [4] 3] with a relatively low "adversarial" threshold for initial assessments and corrects for false negatives using image preprocessing. Our method involves a Convolutional Neural Network (CNN) with dropout which reports the Bayesian uncertainty of its classifications, but which then takes multiple crops of images which fall near our threshold uncertainty level and classifies each crop again using our CNN. The final classification or 


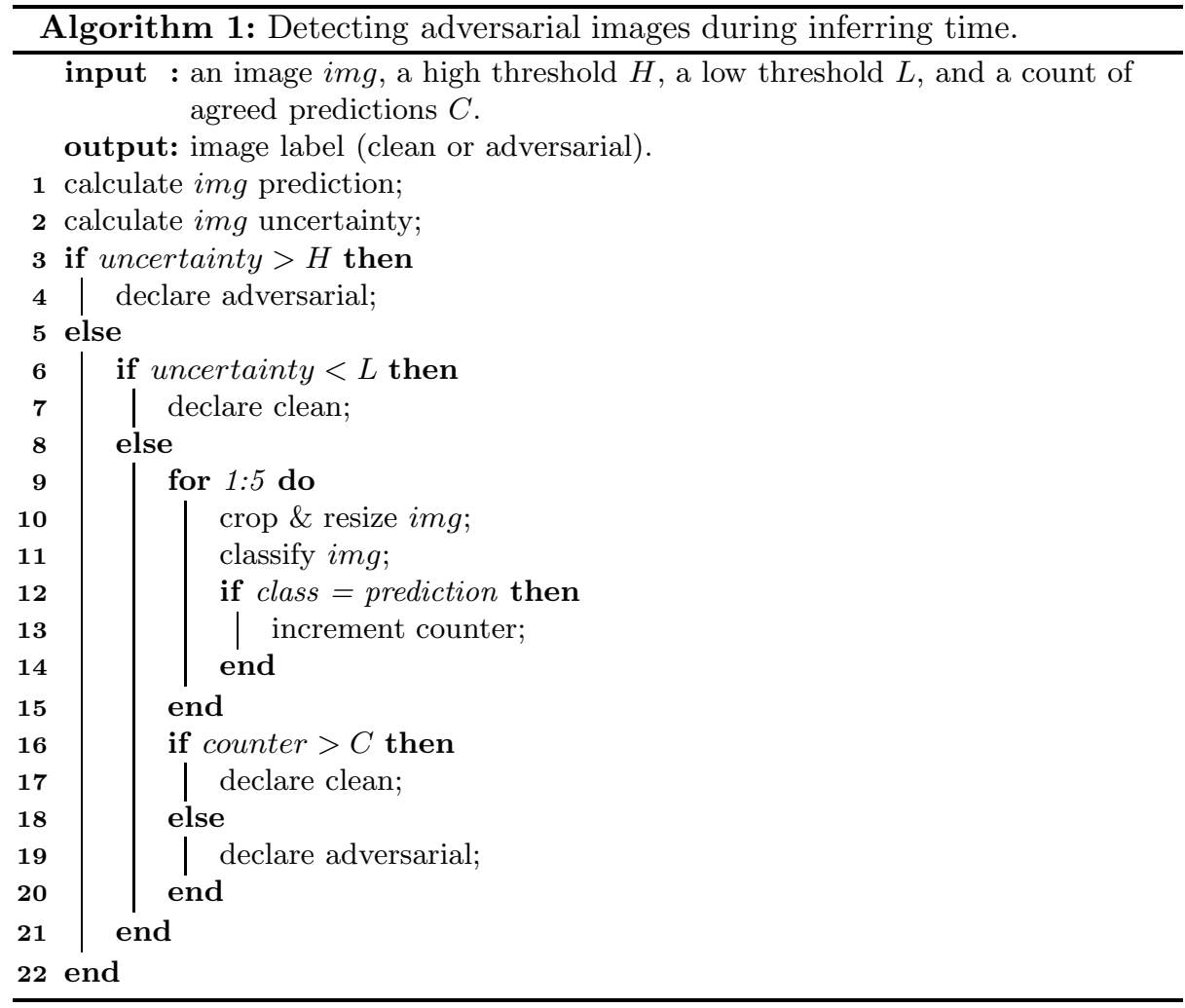

adversarial image indication is based on both the level of Bayesian uncertainty and the agreement or lack thereof among the crops. Algorithm 1 illustrates the procedure.

\subsection{Computing Model Bayesian Uncertainty}

Dropout was first introduced as a means of avoiding overfitting in deep neural network training [17]. Dropout layers mean that some weights are randomly zeroed out, that is, some links between neurons are cut. Gal and Ghahramani 4. noted that including dropout layers in a neural network provides information about uncertainty for a wide variety of DNN architectures, sometimes without modification. Srivastava et al. [17] used dropout at training time, however Gal and Ghahramani applied dropout to the inference stage.

Our model uses dropout layers in the inference stage: For each image, we made $N$ stochastic passes through the network. Each pass produced a probability for each available class by applying Softmax to the resulting logit vectors $z_{1}(x), \ldots, z_{N}(x)$.

To obtain the stochastic prediction, we take the mean of the logit vector $z(x)$ for each class. The image is provisionally classified as the class with the highest 
mean. We also obtain the stochastic uncertainty by computing the standard deviation of the predictions over the $N$ stochastic passes.

\subsection{Image Preprocessing}

At this point, if the stochastic uncertainty is sufficiently high, our model declares the image adversarial without reference to the secondary method. If the uncertainty is sufficiently low, our model outputs the provisional classification as the final classification, again without reference to the secondary method. However, if the uncertainty lies within a certain middle range, the model employs the crop-resize secondary method.

In the cropping stage, the network takes any image which has been classified with an uncertainty laying in the "edge" range, splits it into five overlapping crops (top left, bottom left, top right, bottom right, and center), in the manner of Graese et al. [6], and resizes each crop back to the original size. Each of these crops is reclassified by the network, and if four out of the five new classifications agree with the original, the image is not declared adversarial and the original classification is output. This allows us to set the "threshold" Bayesian uncertainty level low enough to risk a noticeable number of false negatives (clean images declared adversarial) at the uncertainty stage of the process, while providing a mechanism to prevent these "extra" false negatives from making a large impact on our model's final accuracy. The lower uncertainty threshold level, in turn, gives a greater chance of detecting borderline adversarial images.

\section{Experiments}

We implemented our model using Python 3.6 with CleverHans 2.0, TensorFlow, Keras 2.0, Scikit-learn, OpenCV, and Pillow 5.1.0. CleverHans facilitates the generation of adversarial examples using a variety of techniques. Using multiple techniques to generate a set of adversarial images for testing allows us to assess the general applicability of our defense. We trained and tested our model using the MNIST dataset: a dataset of handwritten digits consisting of 60,000 training examples and 10,000 test images. We tested our model with 50/50 mixed clean and adversarial test sets for each of three attacks.

\subsection{Neural Networks}

For MNIST, we used the LeNet [11] convnet architecture. We used a dropout rate of 0.5 after the last pooling layer and after the inner-product layer, as in [3]. On normal, non-adversarial samples, this network shows an accuracy of $98 \%$.

\subsection{Adversarial Attacks}

We evaluated our model using three different methods of generating adversarial images: FSGM [5], BIM [9], and JSMA [15]. 
Table 1. Classification accuracy of undefended model.

\begin{tabular}{l|l|l|l}
\hline & FGSM & JSMA & BIM \\
\hline Classification accuracy & $59.0 \%$ & $52.0 \%$ & $50.0 \%$ \\
\hline
\end{tabular}

Table 2. Results for adversarial image detection.

\begin{tabular}{l|l|l|l}
\hline & FGSM & JSMA & BIM \\
\hline False negative & 252 & 85 & 249 \\
\hline False positive & 134 & 74 & 104 \\
\hline True negative & 4796 & 4856 & 4826 \\
\hline True positive & 4725 & 4892 & 4728 \\
\hline Detection accuracy & $96.1 \%$ & $98.3 \%$ & $96.4 \%$ \\
\hline
\end{tabular}

Table 3. Results of applying the defense with different attacks using MNIST dataset.

\begin{tabular}{l|l|l|l|l|l}
\hline \multirow{2}{*}{ Attack } & \multicolumn{2}{|l|}{ Clean images reported clean } & \multicolumn{2}{|l|}{ Adv. images reported clean } & Classification \\
\cline { 2 - 5 } & $\begin{array}{l}\text { Classified } \\
\text { correctly }\end{array}$ & $\begin{array}{l}\text { Classified } \\
\text { incorrectly }\end{array}$ & $\begin{array}{l}\text { Classified } \\
\text { correctly }\end{array}$ & $\begin{array}{l}\text { Classified } \\
\text { incorrectly }\end{array}$ & Accuracy \\
\hline FGSM & $97.3 \%$ & $0.0 \%$ & $1.7 \%$ & $1.0 \%$ & $99.0 \%$ \\
\hline JSMA & $98.5 \%$ & $0.0 \%$ & $1.4 \%$ & $0.1 \%$ & $99.9 \%$ \\
\hline BIM & $97.8 \%$ & $0.0 \%$ & $1.2 \%$ & $1.0 \%$ & $99.0 \%$ \\
\hline
\end{tabular}

\section{Results}

For our model, we adjusted the uncertainty levels based on the information gain to improve accuracy and used these thresholds to calculate high and low levels of Bayesian uncertainty. Our experiments also showed that requiring four out of five crops be in agreement produced the greatest ultimate accuracy. We generated adversarial images using FGSM, BIM, and JSMA, with separate test runs for each attack.

We tested our model with a set of 10,000 images comprised of $50 \%$ clean and $50 \%$ adversarial images. After testing with the basic, undefended CNN, we removed clean images which were misclassified; in testing the defended model, we used only adversarial images generated from images which were correctly classified by the basic CNN when clean.

For the FGSM attack, as seen in Table 1, our basic CNN without defense had an accuracy of $59 \%$ on the $50 / 50$ mixed test set; most of adversarial images were indeed misclassified. By adding our defense to the network, we were able to detect adversarial images with an accuracy of $96.1 \%$ which increased classification accuracy, for the images flagged as clean, to $99.0 \%$ (see Table 2 and 3 ).

Adversarial images produced using the BIM attack were even more successful against the unprotected CNN, which was only $50 \%$ accurate on the mixed test set. With our defense added, our model handled a BIM mixed test set with an accuracy detecting adversarial images of $96.4 \%$ and a classification accuracy on clean-flagged images of $99.0 \%$. 
On a mixed test set produced with the JSMA attack, our basic CNN had an accuracy of $52 \%$. With our defense, our model achieved $99.9 \%$ classification accuracy with $98.3 \%$ detection accuracy.

In all cases above, accuracy was calculated as <images correctly classified $>$ / $<$ total remaining (flagged as clean) images $>$, and did not include images discarded as adversarial. Detection accuracy was calculated using the $<$ true positive + true negative $>/<$ total images in set $>$. Total images now are less than 10,000 due to removal of misclassified clean images from consideration.

Feinman et al. 3] used a Bayesian uncertainty model, but combined it with kernel density rather than preprocessing. With MNIST, they achieved mixed test set adversarial sample detection ROC-AUC scores of $90.57 \%$ for FGSM, $97.23 \%$ for BIM-A, and $98.13 \%$ for JSMA, slightly lower than the detection accuracy results for our model. On average, Feinman et al. achieved an MNIST detection ROC-AUC of $95.31 \%$, somewhat lower than our average MNIST detection accuracy of $\mathbf{9 7 \%}$.

Graese, Rozsa, and Boult [6] tested their techniques with the FGSM attack, but did not consider BIM nor JSMA attacks. For their 5-crops-and-resize method they reported a classification accuracy of $90.94 \%$ for FGSM, noticeably lower than our combined model results of $\mathbf{9 9 . 0 \%}$ for that attack, however their binarization defense had a comparable accuracy of $99.21 \%$.

Wang et al. [18] applied a set of mutations, obtained by imposing a minor random but realistic perturbation on the image. Based on their observation, clean images preserved their labels while adversarial did not. They scored, on average, 88.0\% for MNIST detection. Ma et al. 12 used Local Intrinsic Dimensionality (LID) to characterize the dimensional properties of adversarial subspaces or adversarial regions which facilitates the distinction of adversarial examples. For MNIST, on average, they scored $96.20 \%$ accuracy, slightly lower than our average.

\section{Conclusion}

Our combined Bayesian uncertainty and image pre-processing proved effective, with accuracy in the high 90s, at detecting adversarial examples in a mixed MNIST test set. This allowed for considerably higher accuracy in classifying the remaining, primarily clean, images. Our model has shown results which are for the most part similar to those of other recent work, with slightly greater accuracy in adversarial example detection for the test sets and attacks we considered.

Future work in those areas could be promising, as our results suggest that a combined defense has some advantages. In particular, it would be interesting to explore a combination method that involves binarization in the manner of Graese et al. Also, future work in defenses against additional attack methods could shed light on the general applicability of this combined method. 


\section{References}

1. Carlini, N., Wagner, D.: Adversarial examples are not easily detected: Bypassing ten detection methods. In: Proceedings of the 10th ACM Workshop on Artificial Intelligence and Security. pp. 3-14. ACM (2017)

2. Carlini, N., Wagner, D.: Towards evaluating the robustness of neural networks. In: 2017 IEEE Symposium on Security and Privacy (SP). pp. 39-57. IEEE (2017)

3. Feinman, R., Curtin, R.R., Shintre, S., Gardner, A.B.: Detecting adversarial samples from artifacts. arXiv preprint arXiv:1703.00410 (2017)

4. Gal, Y., Ghahramani, Z.: Dropout as a Bayesian approximation: Representing model uncertainty in deep learning. In: International Conference on Machine Learning. pp. 1050-1059 (2016)

5. Goodfellow, I.J., Shlens, J., Szegedy, C.: Explaining and harnessing adversarial examples. In: International Conference on Learning Representations (2015)

6. Graese, A., Rozsa, A., Boult, T.E.: Assessing threat of adversarial examples on deep neural networks. In: Machine Learning and Applications (ICMLA), 2016 15th IEEE International Conference on. pp. 69-74. IEEE (2016)

7. Guo, C., Rana, M., Cisse, M., van der Maaten, L.: Countering adversarial images using input transformations. In: International Conference on Learning Representations (2018)

8. Krizhevsky, A., Hinton, G.: Learning multiple layers of features from tiny images. Tech. rep., Citeseer (2009)

9. Kurakin, A., Goodfellow, I., Bengio, S.: Adversarial machine learning at scale. In: International Conference on Learning Representations (2017)

10. LeCun, Y.: The MNIST database of handwritten digits. http://yann.lecun.com/exdb/mnist/ (1998)

11. LeCun, Y., Boser, B., Denker, J.S., Henderson, D., Howard, R.E., Hubbard, W., Jackel, L.D.: Backpropagation applied to handwritten zip code recognition. Neural computation 1(4), 541-551 (1989)

12. Ma, X., Li, B., Wang, Y., Erfani, S.M., Wijewickrema, S., Houle, M.E., Schoenebeck, G., Song, D., Bailey, J.: Characterizing adversarial subspaces using local intrinsic dimensionality. In: International Conference on Learning Representations (2018)

13. Netzer, Y., Wang, T., Coates, A., Bissacco, A., Wu, B., Ng, A.Y.: Reading digits in natural images with unsupervised feature learning. In: NIPS workshop on Deep Learning and Unsupervised Feature Learning. vol. 2011, p. 5 (2011)

14. Papernot, N., McDaniel, P.: Extending defensive distillation. In: IEEE Symposium on Security and Privacy (2017)

15. Papernot, N., McDaniel, P., Jha, S., Fredrikson, M., Celik, Z.B., Swami, A.: The limitations of deep learning in adversarial settings. In: IEEE European Symposium, Security and Privacy (EuroS\&P). pp. 372-387. IEEE (2016)

16. Rozsa, A., Rudd, E.M., Boult, T.E.: Adversarial diversity and hard positive generation. In: Proceedings of the IEEE Conference on Computer Vision and Pattern Recognition Workshops. pp. 25-32 (2016)

17. Srivastava, N., Hinton, G., Krizhevsky, A., Sutskever, I., Salakhutdinov, R.: Dropout: a simple way to prevent neural networks from overfitting. The Journal of Machine Learning Research 15(1), 1929-1958 (2014)

18. Wang, J., Sun, J., Zhang, P., Wang, X.: Detecting adversarial samples for deep neural networks through mutation testing. arXiv preprint arXiv:1805.05010 (2018) 